\title{
Twenty-five questions for string theorists
}

\author{
P Binétruy ${ }^{1}$, G L Kane ${ }^{2}$, J Lykken $^{3}$ and B D Nelson ${ }^{4}$ \\ ${ }^{1}$ LPT, Université Paris-Sud, Bat. 210, 91405 Orsay CEDEX, France \\ 2 Michigan Center for Theoretical Physics, Randall Laboratory, University of Michigan, \\ Ann Arbor, MI 48109, USA \\ 3 Theoretical Physics Department, Fermi National Accelerator Laboratory, Batavia, \\ IL 60510, USA \\ ${ }^{4}$ Department of Physics and Astronomy, University of Pennsylvania, Philadelphia, \\ PA 19103, USA
}

E-mail: bnelson@sage.hep.upenn.edu

Received 14 October 2005

Published 8 December 2005

Online at stacks.iop.org/JPhysG/32/129

\begin{abstract}
In an effort to promote communication between the formal and phenomenological branches of the high-energy theory community, we provide a description of some important issues in supersymmetric and string phenomenology. We describe each within the context of string constructions, illustrating them with specific examples where applicable. Each topic culminates in a set of questions that we believe are amenable to direct consideration by string theorists, and whose answers we think could help connect string theory and phenomenology.
\end{abstract}

\section{Introduction}

Today there is a renewed interest in phenomenology among some part of the string theory community; this despite the fact that sometimes string theorists state that string theory is too poorly understood to try to connect it to the real world. While we would not disagree with that, we think that the needed understanding is more likely to emerge if string theorists take up the study of certain phenomenologically relevant questions. We are hopeful that proposing several questions can encourage string theorists to solve them, or at least formulate them more precisely, and thereby generate closer connections between phenomenological theory and string theory.

The relevance of many of the phenomenological issues we choose to consider will be obvious to even the most formal of theorists, though the relevance of some others may not be as clear. We have chosen to introduce each question (or set of questions) with a discussion of the low-energy physics issues that motivate it. We then attempt to embed this discussion in string theory, often by means of examples from both older and more recent constructions. As we are often reminded by string theorists, the exact nature of string theory is not yet fully 
known. Thus a series of examples may not indicate a theorem about all of M-theory-or even about a particular class of constructions. But we hope they will serve to illustrate the reason for our interest in these topics and stimulate thinking in a phenomenological direction more generally.

The questions fall naturally into several groups. We have chosen to list them in order of increasing apparent difficulty. By this we mean that for some questions the avenue of approach from string theory is somewhat clear, while for others it is more obscure. The most difficult questions will be those that pertain most directly to the low- and intermediate-energy world, in particular those that involve some level of dynamical symmetry breaking. It is often said that such field-theory dynamics is by definition not the subject of study for string theorists. But such thinking is counter-productive: the dynamics of a field theory are often determined by the boundary conditions we apply at the string energy scale, and these boundary conditions are most certainly in the domain of string theory.

There are of course a number of additional questions that can be posed beyond the ones contained here, and we encourage others to suggest them. When questions overlap, but are logically distinct, we have not tried to consolidate them; redundancy might be helpful. Furthermore, there are areas that one would call 'phenomenological' that formal theorists are actively pursuing: obtaining $N=1$ supersymmetry or three generations in the low-energy theory, attacking the cosmological constant problem, issues of early universe physics, moduli stabilization, and so on. These are crucial topics, but as our goal is to stimulate thinking in directions that have been somewhat overlooked, we will not consider them here.

Partial answers to some questions are already known to some people. But these answers are generally not known in forms useful to promote phenomenological progress. We urge experts to publicize results that broaden understanding along the lines we discuss, and in additional areas not covered here as well.

Finally, we do not intend to write a review of open issues in string phenomenology, nor of supersymmetric phenomenology more generally ${ }^{5}$. Thus, after some thought we have tried to provide only a brief discussion, with limited references to work that can be considered relevant to the questions, so as to not dilute the impact of a short, precise paper. Those who answer the questions will provide the references. Much of what follows will be familiar to many, but many reviews of supersymmetric and string phenomenology are available for further elucidation [2-6].

\section{Gauge symmetries}

\subsection{Large groups}

A basic question of phenomenological importance is the rank of the gauge group that results in four dimensions from compactification of the string theory. Symmetries have historically been both our primary means of modelling known phenomena as well as our primary tool for building theories of the unknown. From the string perspective we expect many such symmetries that are operative in the low-energy world to have an origin as a gauge symmetry. The set of models and mechanisms which rely on some new extension of the standard model gauge group is large, and utilizing several in a complete model can quickly put strong demands on the size of the gauge group descending from string theory.

As an example, we might start with the standard model and its rank-4 gauge group $G_{\mathrm{SM}}=S U(3) \times S U(2) \times U(1)$. Achieving a group of this size is quite elementary for most string constructions. But phenomenologists often add much more to this basic structure. For

5 Indeed, an excellent one already exists, in a format similar to this one [1]. 
example, a gauged $U(1)_{B-L}$ can be extremely useful in insuring an intact R-parity, and hence the necessary stability of the proton and/or a cold dark matter candidate. Similarly, if we wish to generate an effective $\mu$-term from the vev of some field we may need yet another $U(1)$ factor. And if we wish to generate Majorana masses for neutrinos we may need yet another. We might also wish to introduce so-called 'horizontal' or 'family' symmetries that operate on the generation indices of the standard model fields in order to generate certain Yukawa coupling patterns. While the simplest of these could be just a single $U(1)$ factor, more realistic models often call for higher rank flavour symmetries, either as products of $U(1)$ factors or as non-Abelian groups such as $S U(2)$ or $S U(3)$ [8-11]. Thus it is not unimaginable that a gauge group with rank 7,8 or even larger might be the best candidate for describing the standard model degrees of freedom.

On top of this we must consider the issue of supersymmetry breakdown. It is natural to consider gaugino condensation as a candidate mechanism in the string theory context. While in principle any asymptotically free gauge group will suffice (notably not one of the groups we mentioned in the previous paragraph), in practice achieving the right scale of SUSY breaking in the observable sector often requires a group of fairly substantial size, such as $S U(5)$ or higher [12]. If we wish to use only the tree-level Kähler potential for the dilaton we must employ multiple condensates in the hidden sector with beta-functions that are tuned against one another. Examples of such configurations with combined rank less than 8 are rare. Indeed, the typical rank of the combined condensing group often needs to be $\mathcal{O}(10)$ - even larger than 16 in many cases [13].

One might argue that accommodating all of this rank is not a problem in string models. But specific string constructions often involve a limit on the allowed rank of the low-energy gauge group. For example, standard constructions of the weakly coupled heterotic string (WCHS) give rise to gauge groups at the (4D) string energy whose rank can be no larger than 16. If one were to allow some or all of the compact spacetime coordinates to be associated with the gauge group, as in the free-fermionic or asymmetric orbifold constructions, this rank could (in principle) be as high as 22 at the string scale. However, it could also be much lower: reduction in rank between the higher-dimensional theory and the 4D theory can occur in compactification through the vacuum values of continuous Wilson lines which act as adjoint Higgs representations. And realizing the gauge group at higher affine level in the underlying conformal theory results in a reduction in the rank of the gauge group as seen from the 4D theory.

This tension between phenomenological demands and the mathematical consistency of the string theory is not confined to the heterotic region of the M-theory space. Semi-realistic (and supersymmetric) models of particle physics involving type-I and type-II string theory have thus far been constructed mainly on toroidal orbifolds or orientifolds, typically involving intersecting $D$-branes. In these cases mathematical consistency of the theory restricts the number of such branes - and hence the rank of the gauge group(s) that can appear on themto 16 or less. It is certainly possible to modify these conditions so as to achieve larger rank, perhaps arbitrarily large. But to date no such construction with a semi-realistic gauge group and spectrum exists without abandoning supersymmetry in the low-energy theory. While the demands of phenomenologists on such models continue to push for larger rank ${ }^{6}$, these models may (in fact) be even more constraining than the WCHS. Achieving sufficient intersection numbers to obtain three families of chiral matter often restricts the allowed rank of the resulting gauge group. Including non-trivial magnetic flux generally restricts the rank

6 For example, the 'racetrack inflation' model based on Type-IIB string theory of [14] wanted two asymptotically free gauge groups with ranks of $\mathcal{O}(10)$, and possibly as large as $\mathcal{O}(100)$. 
even more - perhaps fatally by eliminating candidate hidden sector gauge groups for gaugino condensation [15]. Recent surveys of the string theory landscape for flux compactifications of type-IIB string theory suggest that small rank gauge groups are, indeed, generally preferred $[16,17]$. Faced with this sort of evidence, we pose the question:

Question 1: What are the properties of string constructions that can provide realistic observable sectors while simultaneously providing large-rank gauge groups in a hidden sector?

Where specific mechanisms for alleviating this tension between mathematical consistency and phenomenological necessity have already been suggested (for example, the inclusion of anti- $D$-branes, as in $[18,19]$ ) we would further ask:

Question 2: Can general relations between the mechanism(s) that relax restrictions on the rank of the low-energy gauge group, and the overall low-energy phenomenology of the construction, be identified?

\subsection{Gauge coupling unification}

It has now been more than 20 years since the remarkable observation was made [20-22] that if (a) the hypercharge generator is given a normalization consistent with its inclusion in an $S U(5)$ group, (b) the standard model field content is extended to a supersymmetric model in the minimal manner, (c) these superpartners have masses near the electroweak scale and (d) there are no additional fields in the theory charged under the standard model gauge group to some high-enough energy scale, then the renormalization group (RG) evolution of the measured gauge couplings of the standard model within this paradigm leads to a unification of the three couplings at a very high-energy scale to a very good degree of accuracy. The intervening 20 years of measurements of standard model phenomena have done nothing to weaken this stunning fact - in fact, more precise data have only made the conclusion stronger [23]. Hence the heavy focus of the theoretical community is on low-energy supersymmetry.

While it has become somewhat fashionable of late to take the implicit (if not explicit) view that this apparent unification is merely a coincidence, a more careful study shows that it would have to be a very great coincidence indeed. Even including the uncertainty in superpartner masses (but keeping them within a window near $1 \mathrm{TeV}$ ), the locus of points in the $\alpha_{3}\left(M_{Z}\right)-\sin ^{2} \theta_{w}\left(M_{Z}\right)$ plane consistent with unification at any scale is very small. The fact that the measured values of these two parameters fall within this locus of points was estimated by the authors of [24] to have a chance of occurring in the $(0.2-2) \%$ range if it were merely a coincidence.

It has often been said that gauge coupling unification is a 'prediction' of string theory, but this is somewhat misleading. While it is true that eventually all coupling constants can be related to the string coupling $g_{s}$ and string tension $\alpha^{\prime}$, that does not imply that the 4D theory just below the string scale exhibits a unification of 4D gauge couplings. The relation between the (unified) string coupling and the various low-energy couplings appearing in the effective field theory in four dimensions involves the vacuum expectation value of various moduli associated with the string compactification. Even in the WCHS, where this relation is universal for all gauge groups at the leading order, threshold corrections can affect this leading-order unification and possibly spoil the 'prediction' of gauge coupling unification.

Despite this caveat the compatability of the WCHS with the idea of high-scale gauge coupling unification was held as a major phenomenological success of this theory. Yet in recent approaches where the standard model is generated by overlapping and/or intersecting $D$-brane configurations gauge coupling unification is not at all automatic unless the gauge 
groups of the standard model are all embedded on one stack of $D$-branes. And just as in the WCHS case, threshold corrections from heavy string modes will affect the various couplings of the four-dimensional gauge groups differently, unless a grand unified theory with a simple GUT group exists below the string scale. These threshold corrections involve a host of moduli associated with the compactification itself and will take different forms depending on the type of theory.

Given the apparent challenges for even the WCHS in explaining the apparent unification of gauge couplings at a scale $\Lambda_{\mathrm{GUT}} \sim 10^{16} \mathrm{GeV}$-let alone achieving the correct normalization of hypercharge and the absence of exotic states that ruin the RG evolution [25] — we might well first ask the question:

Question 3: If the apparent gauge coupling unification is not a coincidence, is this alone evidence for the existence of a unification-scale GUT?

One would immediately think the answer is negative, given the fact that plenty of examples can be constructed that do not involve GUTs. For example, it was shown in [26] that heterotic string theory allows for unification at the string scale provided certain geometrical moduli and Wilson line moduli acquire particular vevs that need not be large. But question 3 is asking whether gauge coupling unification, at the scale and to the accuracy inferred from low-energy measurements, could ever be considered a natural or generic outcome in a string model without an operative GUT theory below the string scale and apart from any special configuration of moduli vevs. That is:

Question 4: What are the stringy conditions that would guarantee gauge coupling unification occurs rather than just imposing it?

Thus even if there is no grand unification into a simple group the gauge coupling unification could still be natural if the sizes of the moduli expectation values are about the same or related by some symmetry. The latter may be the only hope that brane constructions can be made compatible with gauge coupling unification. A particularly valiant effort in this direction was that of [27] in which the geometrical properties of a particular type-IIA orientifold construction with $D_{6}$-branes were exploited, along with an assumption about certain moduli expectation values (namely, that the volumes of certain 3-cycles that these branes wrap are equal) to argue that gauge couplings should unify. Even here only one of the two conditions needed for unification becomes satisfied. It is not clear that what could imply the second condition. Certainly the challenge of explaining gauge coupling unification in open string models will require such an interplay of compactification geometry, brane configuration and symmetries among moduli. What we seek is an explanation that can span several classes of brane constructions, so we ask:

Question 5: What are the necessary or sufficient conditions that brane constructions must have for automatic gauge coupling unification to be the result?

\subsection{Hypercharge normalization}

A related, but logically distinct, question that impinges on gauge coupling unification is the issue of hypercharge normalization. Let us take a well-known example to illustrate the problem. In the weakly coupled heterotic string with gauge group $E_{8} \otimes E_{8}$, compactified on an orbifold, we expect nonzero Wilson lines to break the gauge group of the underlying string theory to a product of gauge groups, including a number of $U(1)$ factors. It is a highly nontrivial task to decide which linear combination of these $U(1)$ factors should be identified 
as the hypercharge of the standard model (see section 3.1 below). In fact, the process of isolating the trace anomaly over $U(1)$ factors to one linear combination generally implies mixing among Abelian factors from the observable $E_{8}$ and the hidden $E_{8}$ group, obscuring any residual knowledge of the underlying $E_{6}$ or $S O(10)$ structure. Finally, cancellation of the Fayet-Iliopoulos $D$-term associated with the anomalous $U(1)$ generally results in the breaking of one or more additional $U(1)$ factors, suggesting that identifying the correct hypercharge combination can only be done after model-dependent field theory dynamics occurs. Once this process is carried out, it is typically the case that the hypercharge normalization relative to the other generators of the standard model gauge group is not that which would result from a grand unified group, i.e. $k_{Y} \neq 5 / 3$.

We have illustrated the issue of hypercharge normalization with an example of the heterotic string on a toroidal orbifold, but intersecting brane configurations in open string theories are no less problematic. One may hope to understand a normalization consistent with $S U(5)$ unification in the context of the WCHS since the standard embedding results in an $E_{6}$ gauge factor prior to the introduction of Wilson lines. But in $D$-brane constructions the starting gauge group is generally a product of $U(N)$ factors, without any point in the construction at which a unified group could be said to exist. While the normalization of the Abelian factor in the decomposition $U(N) \rightarrow S U(N) \times U(1)$ may be clear, the one factor of such $U(1)$ 's identified as hypercharge emerges as an arbitrary linear combination-the GUT normalization is then understandable only as a coincidence. While the situation improves somewhat in cases where an underlying Pati-Salam $U(4) \times U(2)_{L} \times U(2)_{R}$ structure is employed, the fact remains that due to the lack of universal gauge coupling determination (see section 1.2 above), hypercharge normalization must still be seen as a fortuitous accident of moduli dynamics. An easier solution is to seek out models that guarantee $k_{Y}=5 / 3$ :

Question 6: What are the necessary or sufficient conditions that would guarantee the normalization $k_{Y}=5 / 3$ for the $U(1)$ factor associated with hypercharge in each class of string constructions?

Even in the absence of such a mechanism the observed high-energy unification of coupling constants could be the result of additional matter with the appropriate charges introduced at particular intermediate scales ${ }^{7}$. In this sense it is possible that the so-called 'exotics,' which are usually considered a nuisance could turn out to be a virtue. Thus we might seek a second-best solution:

Question 7: Are there any reasons to believe that particular compactifications might automatically produce extra states with the right properties to maintain gauge coupling unification even with $k_{Y} \neq 5 / 3$ ?

\subsection{Gauge singlets as matter fields}

One of the most often-employed tools of the model builder is the gauge singlet: by eliminating all possible gauge interactions it becomes easier to solve certain problems in isolation. Examples include the inflation and curvations of early universe cosmology fields to generate neutrino Majorana masses (indeed often the neutrinos themselves - see below), fields to generate $\mu$-terms, fields to drive phase transitions in the early universe, etc. Some of these solutions work less efficiently if these fields carry quantum numbers for some other gauge groups beyond those of the standard model.

7 For a discussion of this, and other issue of gauge unification in string models, we refer the reader to [25] and references therein. 
In string theory, if we classify all 4D fields as either moduli or matter fields, and then agree to define a field as 'matter' if it carries quantum numbers under some gauge interactions, then by implication all such fields described above would have to be moduli. This common rule of thumb about matter and gauge interactions is supported by the dimensional reduction of gauge interactions from ten-dimensional supergravity to four dimensions. Matter states and their superpotential couplings in 4D are then seen as remnants of the 10D super-Yang-Mills interaction, and hence always involve gauge-charged matter.

But the term 'modulus' has a more precise technical meaning as a field with no classical potential — a field which parameterizes the degeneracy of the vacuum of the string theory. Many examples of moduli that are familiar to string model builders fill both the technical definition (no classical superpotential couplings) and the common definition (no gauge interactions). These include the moduli that arise from dimensional reduction of the tensors $g_{M N}$ and $b_{M N}$ of ten-dimensional supergravity, and the dilatonic scalar. Yet many other fields which also parameterize the degeneracy of vacua in a particular string construction do not obey one or the other of the two definitions given above. For example the degrees of freedom that parameterize continuous Wilson lines can be represented as gauge-charged matter [28-30]. In toroidal orbifolds states with a non-zero oscillator number and gauge interactions can be thought of as would be blowing-up modes that parameterize the transition from a singular (orbifold) manifold to a smooth (Calabi-Yau) one [31-33].

In both of these cases the above-mentioned gauge-charged fields have a second role that is moduli-like in nature (i.e. they parameterize a property of the compactification geometry) and typically have superpotential couplings. But fields without gauge charges that might otherwise be unambiguously called moduli can behave as matter as well. Consider, for example, the case of vector-bundle moduli in heterotic string theory compactified on a CalabiYau threefold. The elements of $H_{1}($ End $V)$ for the bundle $V$ with structure group $G$ will be singlets of the gauge group that is the complement to $G$ in $E_{8}$. For example, if $V$ is the $S U$ (3) tangent bundle in the standard embedding, the elements of $H_{1}($ End $T)$ will be singlets of $E_{6}$. The speculation that these fields might couple linearly to gauge-charged $\mathbf{2 7}$ and $\overline{\mathbf{2 7}}$ 's in the superpotential was put forward some time ago [34]. However, in the standard embedding the vector-bundle moduli and geometrical moduli are identified, and any possible superpotential couplings would be severely constrained. More recent work that goes beyond the standard embedding in constructions with $G=S U(4)$ has shown that the vector-bundle moduli, now liberated from their geometric brethren, do indeed couple linearly in the superpotential as has been demonstrated by explicit construction [35]. Yet even the relatively mundane geometrical moduli that characterize the gross shape of the compact space can be thought of as having polynomial superpotential couplings to gauge-charged matter if one is in a regime of moduli space where a suitable expansion of the moduli-dependent Yukawa couplings is available.

Thus it seems that the distinction between 'matter' and 'moduli' is somewhat blurred. Perhaps an inappropriate use of language is to blame, but the issue of true gauge singlets having polynomial superpotential couplings to gauge-charged matter is more serious than mere semantics. If singlet fields couple to charged matter in such a way as to be relevant to low-energy phenomenology then they may fill the roles mentioned at the beginning of this section. But employing true singlets for such tasks as generating Majorana masses for neutrinos or generating a low-energy Higgs bilinear often results in the breaking of unwanted global symmetries with accompanying cosmological difficulties. And singlets with superpotential couplings to standard model fields can destabilize the hierarchy between the electroweak and string scales when supergravity loop effects are considered [37]. Thus it is of great utility to 
have an unambiguous definition of matter fields vis-a-vis moduli fields in string terms, or put differently:

Question 8: Under what conditions can true singlets of all gauge symmetries exist and in what sense can they be truly called 'matter' (i.e. have Yukawa interactions with SM fields)?

As an interesting corollary, let us note that model builders often assume that right-handed neutrinos, introduced to generate neutrino masses, are true singlets themselves. This is made plausible by the fact that they are singlets under the standard model gauge group. But as mentioned above, since we wish these fields to have Yukawa couplings to left-handed doublets it seems unlikely that they can be overall gauge singlets. Nevertheless, if they were we might expect mixing between neutrinos and various string moduli, and such ideas have been suggested in the past [38]. In theories with open-string states a true singlet neutrino might prompt the question of whether neutrinos were in fact open string states or closed string states. Given the difficulty in finding natural manifestations of Majorana mass terms for neutrinos in string models (see section 4.4 below) we might wonder if the unique nature of the neutrino in the standard model particle content is somehow a reflection of something radical:

Question 9: If right-handed neutrinos are true singlets, do they mix with string moduli?

\section{Discrete symmetries}

\subsection{Classifying electroweak doublets}

In the standard model the combination $B-L$, where $B$ is the baryon charge of a field and $L$ is its lepton charge, is a perturbatively conserved global quantum number. When the standard model gauge group arises from the decomposition of $S O(10)$ or $E_{6}$ then $B-L$ can be promoted to a local gauge symmetry and is generated by a linear combination of the various Abelian generators of the decomposition. Interestingly, $B-L$ is one of only two linearly independent additional $U(1)$ factors that can be added to the standard model and be anomaly-free with just the standard model field content. Most importantly, an exactly conserved $B-L$ implies a perfectly stable proton. Thus this particular $U(1)$ factor-or a discrete subgroup of it-holds an important place in low-energy phenomenology.

In this section we wish to focus on lepton number, returning to baryon number below. Most semi-realistic string constructions with $N=1$ supersymmetry, whether of the heterotic or open-string variety, have a spectrum with more fields than those of the MSSM. These 'exotics' often contain $S U(2)$ doublets that are $S U(3)$ singlets. Even when an underlying $S O(10)$ structure was once present, the need for twisted sectors in the superstring spectrum often implies that the states of the low-energy spectrum need not fill out complete $\mathbf{1 6}$ and $\mathbf{1 0}$ representations. Thus an immediate ambiguity ensues: should these exotic fields be classified as Higgs doublets or lepton doublets?

Without $S O(10)$ to guide us, or a gauged $U(1)_{B-L}$ or discrete R-parity which can be embedded in an $S O(10)$ structure, the only way to distinguish between lepton and Higgs doublets is through their superpotential couplings to other quarks and leptons. Yet the notion of lepton doublet versus Higgs doublet may be meaningless from the point of view of string selection rules. While some degree of mixing between the two species of doublet may be tenable for low-energy phenomenology, arbitrary mixing generally is not [39]. Most importantly, proper electroweak symmetry breaking and the absence of massless Pecci-Quinn axions requires a $\mathrm{TeV}$-scale bilinear for just one type of doublets - those that we identify as Higgs states. 
If we must appeal solely to string selection rules and the string physics which determines Yukawa interactions in order to distinguish leptons from Higgs fields, then the structure of the MSSM Lagrangian can only be a fortuitous accident in the sense that not all possible operators are to be allowed. In theories of intersecting $D$-branes the analogue to sectors at various fixed points is the identification of MSSM states with strings localized at the intersection of various branes containing standard model gauge groups. Then $U(1)_{B}$ and $U(1)_{L}$ might individually be understood as arising from the decomposition $U(3) \rightarrow S U(3) \times U(1)_{B}$ and $U(2) \rightarrow S U(2) \times U(1)_{L}$. That is, different sectors of the Hilbert space, determined by geography in the compact space, are correlated with representations under the SM gauge groups, with particle number symmetries understood topologically. This is an appealing way to understand these symmetries in the absence of $S O(10)$ gauge symmetry, but does not fully distinguish leptons from Higgs states-not to mention the problem of generating a Higgs bilinear (see section 4.3 below). Thus a fundamental concern of low-energy phenomenology is the following:

Question 10: Can a definition of lepton number that distinguishes lepton doublets from Higgs doublets be unambiguously defined for string theory in the absence of an underlying $S O(10)$ gauge structure?

\subsection{Proton decay and baryon number violation}

Experimental searches for proton decay will eventually probe lifetimes of about $10^{35}$ years. The longevity of the proton suggests that higher-dimensional operators formed solely from standard model fields must be suppressed by a large mass scale $\left(\gtrsim 10^{16} \mathrm{GeV}\right)$. For supersymmetric theories with a high string scale (i.e. near the Planck scale) such as the ones we imagine here, this requires that all operators of mass dimension four and five which can mediate proton decay must be eliminated from the theory, or at least adequately suppressed. Some set of discrete symmetries is generally assumed to accomplish this task. Of course, if baryon number is related to some gauged symmetry, such as $U(1)_{B-L}$ then such operators can be forbidden if this symmetry is broken only spontaneously by the appropriate vev of some field.

As mentioned above, $D$-brane models provide a framework for understanding the gauging of baryon number via the emergence of the standard model $S U$ (3) factor from a fundamental $U$ (3) factor. But $D$-brane constructions also tend to involve fundamental string scales that are somewhat or significantly below the scale $M_{\mathrm{PL}}=2 \times 10^{18} \mathrm{GeV}$. In many cases a spontaneously broken gauged $U(1)_{B}$ is not enough to protect the proton-some additional discrete symmetry is again needed. It is almost always the case in heterotic models (even those such as free-fermionic constructions based on the NAHE basis set [40, 41] where a 'frustrated' $S O(10)$ symmetry can help forbid some operators in the superpotential) that forbidding dimension four and five operators require particular discrete symmetries.

We will have more to say about these symmetries in the following question, but here our interest is in the dimension six operators, assuming that lower dimensional ones are somehow forbidden to all orders. Generally speaking, operators that mediate proton decay at dimension six arise from the exchange of new gauge bosons that connect quarks and leptons. In many string constructions, particularly heterotic ones, a unified group containing such gauge fields exists prior to the imposition of Wilson line breaking upon compactification. As such, these states are projected out of the light spectrum and (for large string scales) are incapable of inducing proton decay observable in any foreseeable experiment. This conclusion, though drawn after consideration of heterotic models, continues to hold in open string constructions such as type-IIA theory with $D_{6}$-branes [42]. What is most interesting, from a phenomenological point of view, is whether the converse holds, namely: 
Question 11: In a theory where dimension four and five proton decay operators are forbidden, would the observation of proton decay imply the unification (in four dimensions) of quarks and leptons in a simple gauge group, or could observable proton decay arise in such a string theory without grand unification?

\subsection{R-parity}

A related but not identical question is whether there are mechanisms that can be discerned from the string theory level to guarantee the presence of a matter parity, or R-parity, in the lowenergy superpotential. Such an R-parity would do much (but not all) to resolve the issues in the previous question. Furthermore, in the presence of such a parity the lightest supersymmetric particle (LSP) is stable. The stability of the LSP is important enough to warrant its own question (see below), so here we focus specifically on the issue of an intact matter parity such as R-parity.

An intact R-parity without a gauged $U(1)_{B-L}$ generally arises as an accident in string constructions, if at all. To determine if an accidental R-parity exists, a laborious procedure is required. After calculating the spectrum of light states one must compute the allowed Yukawa interactions and then identify the states of the standard model via their leading Yukawa interactions and allowed hypercharge assignments. After this is done, and possible F- and D-flat directions are identified, the effective superpotential terms that survive along each flat direction can be studied to determine the existence of discrete symmetries such as R-parity. Since the standard model gauge group does not forbid R-parity violating terms, constructions that lead directly to the standard model may have such terms. Some may arise at the leading (trilinear) order, and others may arise at higher order in an expansion in $M_{\mathrm{PL}}^{-1}$, presumably reflecting higher-genus terms from the underlying string theory.

These higher-order terms cannot be neglected, for if R-parity is violated, the magnitude of violation must be very small. While certain R-parity violating operators can exist in isolation with $\mathcal{O}(1)$ coefficients, as argued above string models that do not have an intact R-parity tend to allow all such operators at various mass dimensions in the superpotential. A more satisfying solution is to identify R-parity as a symmetry of the underlying compactification geometrysuch as a remnant of a modular symmetry [43]. As the geometry of the compactified space determines the superpotential (both the tree-level and higher-genus terms) it is reasonable to ask the following question:

Question 12: Would any such R-parity be an exact symmetry of the string theory or could it be an approximate parity? If the latter, how large might the violations be?

\subsection{A stable new state}

The longevity of the LSP has profound implications, both for collider phenomenology and for cosmology. In collider events a long-lived LSP, which is presumed to be electrically neutral, exits the detector and the missing transverse energy can be used as a trigger. Many of the most familiar supersymmetry search strategies rely on this trigger for the analysis. Here it is the relevance of a stable LSP for cosmology that we wish to consider.

In cosmology, a stable LSP provides a possible weakly interacting dark matter candidatethough the details of whether such a particle can indeed be the missing non-baryonic dark matter is a model-dependent issue. In contrast, if the LSP has a finite lifetime then it is unlikely to provide the required dark matter. Here we are explicitly talking of the lightest state in the superpartner spectrum and the discrete symmetry protecting its lifetime is presumably the R-parity of the previous question. Other stable or quasi-stable particles could emerge in 
the string theory spectrum and have been suggested in this context: modulinos [44], exotic gauge-charged matter [45], hidden sector matter composites [46], hidden sector gauge composites [47] and wrapped $D$-branes [48]. It could be that one or more of these states (or others not yet imagined) contribute to the dark matter of the cosmos and thus it is appropriate to ask about the stability of new states in a general context:

Question 13: What string theory conditions are sufficient to guarantee stable states beyond the standard model particles?

\section{Flavour and $\mathbf{C P}$}

\subsection{The meaning of flavour}

The problem of understanding the physics behind the masses and mixings of the standard model fermions is a venerable one which has yet to be satisfactorily solved. Yet many approaches to a solution exist, some of which have already been alluded to (see section 1.1 above). Phenomenologists try to understand the hierarchies in fermion masses through symmetries, either discrete or continuous, that act on some flavour index that represents the three states of each species of field in the standard model [49]. Thus it might at first seem that translating these symmetries to the string level is a matter of simply understanding how the triple replication of families arises in string theory. We will have more to say about this translation process in the next question, but there is something more fundamental to be considered first: just what does 'flavour' mean from the string point of view? Let us illustrate the thought process behind this question with an example from heterotic string theory.

When working out the massless spectrum of the bosonic heterotic string on an orbifold we 'tag' different fields by a whole host of properties that are hidden from view in the fourdimensional effective field theory. Here are some examples: untwisted states will carry some amount of H-momentum. Twisted states will be identified by fixed point locations-on the $Z_{3}$ orbifold this means identifying a fixed point location in each of three possible complex planes. Furthermore, there can be twisted fields with non-zero (left-moving or right-moving) oscillator number of some amount, and the direction of this oscillator excitation in the compact space must also be specified. These 'internal quantum numbers', if you will, determine the Yukawa couplings through the string selection rules, so they are 'proto-flavour' in nature.

Thus there is presumably, for every string construction that gives rise to three generations, a natural basis in which to embed the concept of proto-flavour. In some constructions this natural basis is easy to identify. For example, in the $Z_{3}$ orbifold of the bosonic heterotic string it is natural to obtain three generations in the following manner. One postulates the existence of a non-trivial Wilson line in the first two of the three complex planes of the factorizable orbifold. In each of the three complex planes the $Z_{3}$ action leaves three points invariant. A twisted sector state is then labelled in part by its geography: the fixed point location in each of the three planes. Since there are three possibilities in each plane, one naively expects a 27 -fold redundancy in the spectrum, or 27 generations of matter. But the presence of Wilson lines in two of the three planes brings this number to three. Then we might say that (modulo issues of oscillator number) 'proto-flavour' - that is, generation number labels - are naturally defined by fixed point location in the third complex plane.

This may not be completely satisfactory to explain the actual flavour structure of the standard model (see the next question), but it is a natural starting point. In other constructions the path to three-fold replication is less transparent, so the embedding of generation indices in the string quantum numbers is more complicated [50]. Nevertheless a natural embedding should be identifiable. For example, in the free-fermionic models based on the NAHE basis 
set there are nominally 48 generations, but additional projections reduce this to one per basis vector $b_{i}$ [51-53]. These $b_{i}$ are boundary conditions for the free-fermionic fields, specifying the phase they acquire after parallel transport around a noncontractable loop. In the analogous $Z_{2} \times Z_{2}$ asymmetric orbifold construction these three generations are identified with the three possible twist assignments under the two $Z_{2}$ factors.

In open string theories involving intersecting $D$-branes the multiplicity of states is given by the intersection numbers of the cycles wrapped by branes on which these open strings end. Here it is not necessary that each state in the low-energy spectrum receive the same multiplicity. Furthermore, explicit examples may have two 'generations' arising from different intersections from the third-see, for example, the construction in [54]. A rough analogy can be made, however, between states at the intersection of branes at angles and twisted states of heterotic orbifolds, and indeed just such a $2+1$ splitting was engineered in a recent $Z_{6}$ symmetric heterotic orbifold [55]. So once again, the classification of compactifications by their geometrical properties should also give rise to a classification of 'proto-flavour' symmetries, perhaps associated with representations under various twist symmetries, prompting us to ask:

Question 14: Can constructions be classified by the manner in which generation number is embedded in the string-theoretic properties of the light spectra?

\subsection{Selection rules as flavour symmetries}

Let us assume the answer to question 14 is affirmative and a classification of some set of string models is carried out. What we would have for the models in this set is a set of symmetry relations that act on some properly defined generation index at an energy scale just below the compactification scale. This is not yet a true theory of flavour, but it is a suggestive starting point. But unless each generation of matter fields in the MSSM, which fill out complete multiplets of $S U$ (5) (or $S O(10)$ if we include the right-handed neutrino), come from the same sector of the string Hilbert space there is no reason to believe that the embedding described above represents what we mean by flavour in the low-energy sense. That is because at low energies we define flavour by the Yukawa couplings of the light fields. Thus, at some level, the proto-flavour of the string theory must be translated into a theory of flavour as reflected in the labels 'up, down, strange, ...' of the standard model.

What is more, the spectrum that survives to the electroweak scale (and which presumably includes the MSSM particle content) may be quite different from that which exists just below the string scale. In general we expect vacuum expectation values to arise for many fields along various flat directions. Typically this is considered a welcome feature: it may allow for dynamically generated mass terms that project out unwanted 'exotics' from the spectrum while Higgsing unwanted gauge symmetries. But typical string models have many more states with standard model quantum numbers than those of the MSSM. In the $Z_{3}$ example mentioned above typical models may have $\mathcal{O}(10)$ anti-triplets of $S U(3)$ and $\mathcal{O}(20) S U(2)$ doublets. The ones that survive to be labelled $d_{i}^{c}, u_{i}^{c}, L_{i}, H_{u}$ and $H_{d}$ are likely to be linear combinations of states which remain massless along a given flat direction and those that get large masses along the same direction. In such a world the translation of 'proto-flavour' symmetries into flavour symmetries involves a nonlinear mapping.

Should we conclude from the above discussion that any flavour symmetries which might descend from the underlying string theory are likely to be discrete symmetries, or at best Abelian continuous ones? To date these are the only such symmetries that have been identified in explicit string constructions. Such a view point might be supported by considering string selection rules which serve to determine the allowed Yukawa interactions of light fields. Such 
rules are relations that reflect the proto-flavour and ultimately have a geometrical meaning; thus it should be possible to understand them topologically. Their manifestation in the effective field theory just below the string scale is typically in the form of discrete symmetries. So we might first wish to know the answer to the following question:

Question 15: How can string selection rules which determine the superpotential be interpreted as low-energy flavour symmetries?

And then, given that the answer to the above is likely to be easiest for the case of discrete or continuous Abelian symmetries, we ask the obvious follow-on question:

Question 16: Under what circumstances will string-derived flavour symmetries take the form of continuous non-Abelian horizontal symmetries when acting on the low-energy degrees of freedom?

\subsection{Classification of Kähler manifolds}

The Kähler potential is every bit as important as the superpotential in understanding the flavour structure of the low-energy Lagrangian. Both at the tree level and the loop level the elements of the curvature tensor formed by the Kähler potential determine the flavour structure of soft terms and Yukawa couplings, the latter through field redefinitions necessary to achieve a canonical kinetic energy term for the matter fields. Isometries of the Kähler manifold for the nonlinear sigma model describing the light chiral superfields thus have some relevance as flavour symmetries.

The supergravity models typically employed to study the phenomenology of string theories start with a Kähler potential for the matter fields that is diagonal in form

$$
K=f(Z, \bar{Z})_{i}\left|\phi_{i}\right|^{2}+\cdots,
$$

where the fields $Z$ and $\bar{Z}$ represent some set of the string moduli. This is true at least to the leading order calculated from the string theory ${ }^{8}$. The ability to put the leading terms in the Kähler potential into a diagonal form may result from the application of certain isometries (in particular employing modular symmetries) and presumably this diagonal form is in the basis defined by the stringy quantum numbers we called 'proto-flavour' above. Among these string-defined properties are the representation of the fields under the various gauge groups that result from the compactification. For example, in the heterotic string theory on an orbifold the untwisted sector of the theory exhibits such a diagonal structure. Thus it may be natural to expect a form such as

$$
K=f(Z, \bar{Z})_{Q}|Q|^{2}+f(Z, \bar{Z})_{U}\left|u^{c}\right|^{2}+\cdots
$$

to emerge.

Of course we are suppressing the generation, or flavour, indices on these expressions. Even if we had a stringy definition of flavour and it happened to be the case that each individual term in the Kähler potential (2) was also diagonal in the (low-energy) flavour basis we must still sum over many such terms which appear at the same order, not to mention terms which appear at higher order in the string theory. These terms, involving fields with the same standard model quantum numbers, would tend to involve a mixing of flavours that would be incompatible with the diagonal assumption of the Kähler potential. Any such mismatch between the stringy 'diagonal' Kähler basis and the low-energy flavour basis would suggest

\footnotetext{
8 To fully understand the flavour structure of the effective Lagrangian at string energies it is necessary to know the complete curvature tensor. This requires knowing at a minimum the higher-genus terms of the Kähler potential trilinear and quartic in the chiral superfields.
} 
that flavour physics is at least in part arising from the mismatch of fields in the superpotential and the Kähler potential. Again, a topological classification of Kähler manifolds plus a stringy definition of flavour may shed light on this issue. It is this rough idea that we might have in mind when we ask the question:

Question 17: In what basis should we expect the leading-order Kähler potential for massless gauge-charged fields to be diagonal? Under what circumstances might this be a basis that is also diagonal in the low-energy flavour basis?

But merely being diagonal is not completely sufficient to be safe from all constraints from flavour-changing neutral current data, though it is a major step in that direction. It is also necessary for the entries on the diagonal of the scalar mass matrices, which depend on the Kähler metric, to be roughly equal (in the basis in which fermion masses are diagonal). In other words, if these entries are not equal then the super-CKM rotations of the squarks to the quark basis will introduce flavour changing effects proportional to these differences. Thus we need universality in the functions $f(Z, \bar{Z})$ of (1) as well:

Question 18: If the Kähler metric is diagonal in some field basis, under what circumstances should the values of the diagonal entries be equal?

\subsection{Phases and $C P$}

When addressing the relation between string theory and complex phases the most commonly taken strategy is to assume that $\mathrm{CP}$ is violated spontaneously at the field theory level through the (complex) vacuum expectation value of one or more string moduli. Given a concrete moduli stabilization mechanism the values of these complex vevs can presumably be deduced. As the couplings of the various moduli to observable sector fields is known, this should provide a way of understanding the nature of $\mathrm{CP}$ violation in the CKM matrix and/or the soft supersymmetry-breaking Lagrangian.

The ubiquity of this approach is often said to be motivated by the assertion that $\mathrm{CP}$ is a gauge symmetry from the point of view of the string theory and is preserved, perturbatively and even nonperturbatively [56]. Thus it is left to spontaneous breaking of CP, capable of being described in an effective field-theoretic manner, to account for the $\mathrm{CP}$ violation of the observable world. But surely the string theory is not completely mute on this point: we know that symmetry breaking that may appear spontaneous in one context often appears geometric, or explicit, in another context. For example, the study of orbifolds with discrete torsion seems to indicate that Yukawa couplings might acquire a complex phase of a geometric nature, quite apart from the moduli dependence of these couplings, that can be interpreted as an analogue to nontrivial Wilson lines for gauge fields (thus relating a geometrical breaking to a spontaneous breaking) [57]. Furthermore, early in the study of Calabi-Yau spaces it was noted that some Calabi-Yau manifolds might admit an antiholomorphic isometry that could imply (a presumably explicit) violation of CP [58].

Thus it is possible that, prior to any question of moduli stabilization and supersymmetry breaking, a theory of $\mathrm{CP}$ violation and a delineation of the possible physical and allowable phase structure of the effective theory can be formulated already at the string level. Such a formulation would be extremely powerful, either in disfavouring certain classes of models or suggesting others as phenomenologically interesting. In constructions where it can be conclusively demonstrated that $\mathrm{CP}$ violation can only occur through spontaneous symmetry breaking in the low-energy field theory, then it might still be possible to segregate contributions into those related to vevs for the lowest components of chiral superfields (and thus likely to be 
related to effective Yukawa couplings and a theory of flavour) and those related to vevs for the auxiliary fields of the same multiplets (and thus likely related to a theory of supersymmetry breaking and transmission). Such a classification would be a giant step forward in the understanding of CP violation in the observable world. Hence we ask:

Question 19: What are the stringy ways in which complex phases can enter the observable world and can these be related to a theory of flavour or supersymmetry breaking?

\section{Dynamical (super)symmetry breaking}

\subsection{Identifying hidden (and sequestered) sectors}

A principle question of formal string theory is the issue of how many supersymmetries exist in the four-dimensional effective field theory after compactification. This is a topic amenable to string theory consideration in that it involves the determination of the holonomy group of the compact manifold - a geometrical question. But once the theory is compactified, the question of breaking any remaining supersymmetries is then typically relegated to the domain of phenomenology in as much as a field theory explanation (such as gaugino condensation) is often employed. Thus, it is often said, string theory has little to add to the fundamental question of supersymmetry breaking once compactification has occurred.

This argument is overly pessimistic: any particular string construction can be characterized by certain allowed internal geometries and the specific moduli fields that parameterize them ${ }^{9}$. In effective theories derived from string constructions it is often precisely the chiral superfields that represent these moduli that communicate supersymmetry breaking to the fields of the supersymmetric standard model. While the stabilization of these moduliand hence the determination of the exact values of their auxiliary fields-continues to be a difficult problem most profitably addressed by effective field theories, some key features continue to be geometrical in nature. And geometrical properties allow for more robust and generic statements that can help separate classes of models.

The foremost of these features is the existence of 'hidden sectors'. Let us be precise in our definition of this often-used term. A hidden sector, properly speaking, should be one that can communicate to other sectors only via the supergravity multiplet or non-gaugecharged (i.e. singlet) fields with no superpotential of their own, which we will call moduli. If the supergravity multiplet is the only source of communication we will call this special class a sequestered sector model. If gauge fields can participate in the communication of supersymmetry breaking, then this we will call this a 'partially hidden' sector. It is still hidden in the limited sense that a loop diagram is required to communicate the supersymmetry breaking to the standard model supermultiplets. But in some sense the introduction of gauge messengers blurs the geometrical distinction between the sectors, which is our primary interest. Note that direct tree-level communication of supersymmetry breaking ('visible sector' models) is not forbidden, but is usually not considered since in that case one must then contend with the fact that with only the standard model gauge group at least one squark or slepton must be lighter than the corresponding fermions $[59,60]$.

These distinctions are crucial to understanding the nature of SUSY breaking transmission to the sector where the MSSM resides. Low-energy models of SUSY breaking (more correctly, models of the transmission of SUSY breaking to the MSSM) can be crudely divided into

9 By 'string construction' we mean not solely one of the five or six branches of the M-theory amoeba but something more specific, such as one of those branches and a class of compactifications to 4D. For example, weakly coupled heterotic $E_{8} \times E_{8}$ on orbifolds, strongly coupled heterotic $E_{8} \times E_{8}$ with $D_{5}$-branes on a Calabi-Yau, type-II string theory with $D_{6}$-branes on orientifolds, etc. 
those based on mediation by the superconformal anomaly, those mediated by moduli and those mediated by gauge fields. Sequestered sectors can only use anomaly mediation, hidden sectors can use both anomaly mediation and moduli mediation (somewhat misleadingly called 'gravity' mediation in the literature), and a partially hidden sector can use both of these and gauge mediation, and perhaps other mechanisms as well. Given the ubiquity of moduli in string theory a truly sequestered sector may be hard to find. Some studies suggest that even when sectors of the theory can be physically separated in the compact space by a bulk which contains only the gravity multiplet, branes tend to warp the internal geometry and induce (moduli-dependent) supersymmetry breaking [61, 62]. If this finding can be promoted to a theorem then it would be most interesting for phenomenology in as much as the idea of anomaly mediation, with its insensitivity to ultraviolet physics and its automatic solution to the supersymmetric flavour problem, would be strongly disfavoured in string models.

But explicit string constructions of both the heterotic and type-II variety suggest that even hidden sectors may be rare, with most being only partially hidden. For example, the $E_{8} \times E_{8}$ heterotic string has two sectors that are (in principle) hidden from one another, while the $S O(32)$ heterotic string does not-but that is prior to compactification. Once the gauge group is broken by Wilson lines the Abelian factors that arise upon compactification tend to span both $E_{8}$ factors-meaning these sectors are now only partially hidden. The anomalous $\mathrm{U}(1)$ is a prime example, and models have been constructed where the massive gauge bosons of this anomalous U(1) play a role in transmitting SUSY breaking to the observable sector $[63,64]$. Furthermore, states which are bi-fundamental under a subgroup of the standard model and under a hidden sector group have been shown to be present in bosonic heterotic string constrictions [65, 66], free-fermionic constructions [67], type-IIA constructions [54] and others.

This suggests that all three SUSY transmission mechanisms may be present simultaneously, with the only question being which mode dominates. This latter point is a statement about the relative scales of symmetry breakdown in a hidden sector and the mass scale of the suppression factor in the operator that connects this breakdown to the standard model. The bottom line is that the issue of how 'hidden' the hidden sector truly is revolves around the geometry of the manifold of compactification and its corollary: the states of the low-energy massless spectrum. Even the scale of the operators connecting various sectors in the low-energy field theory are set by the string construction being considered. Thus we feel that it is not inappropriate to ask the following question:

Question 20: Can compactifications be considered and classified, at a topological level, so as to identify those that give rise to sectors which are truly hidden and/or sequestered from one another?

\subsection{Gaugino masses}

Whether gaugino soft mass terms are degenerate or not is of great phenomenological importance. For example, if the gaugino masses are degenerate then the complex phases can be rotated away. Moreover, if they are degenerate then light gluinos must imply very light charginos and neutralinos (unless there is a significant amount of extra matter at intermediate scales with special properties), and the latter masses are very constrained by LEP data. Electroweak symmetry breaking can only occur with tuned soft masses in the soft supersymmetry breaking Lagrangian if gaugino masses are degenerate. Finally, collider data are likely to probe and measure the parameters of the gaugino sector of a supersymmetric theory long before usable information on the scalar masses or trilinear scalar couplings is available. 
In heterotic string theories the tree-level gaugino masses are normally degenerate, in that they arise from the auxiliary field of one unified chiral superfield: the dilaton. This property is robust in the sense that it remains true over a wide array of string theories and compactifications, though it need not hold in some open string theories. However, even in the large class of string theories with universal (tree-level) gaugino masses these masses can be suppressed-e.g., if supersymmetry breaking is due mainly to nonzero vevs for the auxiliary fields of various types of moduli associated with the geometry of the compact space, rather than that of the dilaton. Usually the one loop masses are non-degenerate and relatively large when the tree-level masses are suppressed, so the resulting gaugino masses are not degenerate [68].

Although one is discussing soft masses that arise from supersymmetry breaking, we think that one can answer such questions without understanding the precise nature of how supersymmetry is broken by considering classes of supersymmetry breaking. For example supersymmetry breaking can be represented by non-vanishing auxiliary fields for different classes of string moduli. When combined with the moduli dependence of the gauge kinetic function (known in many string constructions both at the genus zero and genus one level) this yields a general structure for gaugino masses in the low-energy theory. From this point the issue of gaugino masses (at least at leading order) is related to the question of gauge coupling unification (see section 1.2 above):

Question 21: In what classes of string theories are tree-level gaugino masses likely to be suppressed?

\subsection{The $\mu$-term 'opportunity'}

One of the most important parameters in the low-energy supersymmetric Lagrangian is the $\mu$ parameter: a supersymmetric mass term which couples the two Higgs doublets of the MSSM. The value and sign of this parameter strongly affects the neutralino and chargino masses, sets the size for the mixing between scalar fermion gauge eigenstates in the mass eigenstate basis and constrains the possible minima of the electroweak Higgs potential. If the value of this mass term was much larger than a $\mathrm{TeV}$ the entire theory would be incompatible with observations. The fact that string theory tends to predict that there are no fundamental bilinears in the superpotential, (i.e. there are no supersymmetric mass terms for the massless spectrum) implies that it must be generated dynamically somewhere below the string energy scale. This is a major step forward in understanding why the $\mu$ parameter is so small.

There are two broad classes of solutions to completing this understanding. One possibility is that the $\mu$ parameter is the result of some dynamical symmetry breaking in which some field $S$, which is a singlet under the gauge factors of the standard model, couples through a superpotential term $W=\lambda S H_{u} H_{d}$ to the Higgs doublets of the standard model. Such a term is common in heterotic string constructions, for example, since the $S$ field can be identified with the singlet under the decomposition $E_{6} \rightarrow S O(10)$ and the requisite coupling is allowed in the $27^{3}$ coupling of $E_{6}$. In many circumstances the necessary vev occurs naturally at approximately $1 \mathrm{TeV}$ through a radiative process not unlike that which drives electroweak symmetry breaking in the MSSM $[69,70]$.

However, a priori we have no reason to expect this mechanism to explain why the $\mu$ parameter should be comparable to the scale of supersymmetry breaking in the observable sector. That it turns out this way often in the case described above appears as a fortuitous accident. In the second class of solutions, which has come to be called the Giudice-Masiero (GM) mechanism [71], this relation between scales is automatic. If a term of the form

$$
K=\frac{1}{2}\left[\alpha_{i j}(Z, \bar{Z}) \varphi^{i} \varphi^{j}+\text { h.c. }\right]
$$


appears in the Kähler potential then an effective supersymmetric mass term will be generated when supersymmetry breaking occurs proportional to the gravitino mass and the function $\alpha_{i j}(Z, \bar{Z})$ plus its derivative.

The two Higgs doublets of the MSSM are unique in being the only vector-like superfields of the MSSM field content. Nevertheless, bilinears in the Higgs doublets are not the only guage-invariant bilinears that can be constructed with the MSSM fields. If a mechanism such as (3) is the source of the $\mu$-term in the low-energy theory then there is likely to be some stringy property of the fields identified as Higgs doublets that allows this term (and only this term) to exist in the Kähler potential ${ }^{10}$. To date there exists precisely one explicit calculation of such terms in the Kähler potential [72], performed in the context of heterotic string theory on a particular orbifold.

It is instructive to consider the peculiarities of this particular case. The existence of a coupling such as $(3)$ required a $(2,2)$ compactification, so that the theory would contain states that transform as both $\mathbf{2 7}$ and $\overline{\mathbf{2 7}}$ representations of $E_{6}$. This is already an interesting fact, as such popular choices as the $Z_{3}$ orbifold do not fall into this category. This suggests that the states we might wish to identity as Higgs states of the MSSM are not those that arise from the 10 representation of $S O(10)$, as is usually assumed. Furthermore, the structure of the function $\alpha_{i j}(Z, \bar{Z})$ involved in the coupling (3) suggests that in this case the doublets involved should be thought of as Wilson line moduli, and that they should transform non-trivially under certain $S L(2, Z)$ symmetries. As such, these states will also appear in the stringy threshold corrections to gauge kinetic functions at one loop in the effective field theory. The important point is that a relation exists between the existence of a desired coupling in the low-energy effective supergravity Lagrangian and many other key properties of the theory: the types of moduli present in the theory and their couplings, the symmetries present in the low-energy theory and the relation between the string properties of various states and their low-energy properties. This suggests that the ' $\mu$-problem' really should be thought of as the ' $\mu$-opportunity', as this issue probes many features of the underlying string theory:

Question 22: What are the requirements on the fields that we wish to consider Higgs states in order to implement the Giudice-Masiero mechanism to generate a $\mu$-term?

Question 23: Are there other string theory (as opposed to field theory) mechanisms which guarantee the relation $\mu \sim m_{3 / 2}$ while simultaneously predicting $\mu \rightarrow 0$ in the supersymmetric limit?

\subsection{Majorana neutrino masses}

The solid experimental evidence of neutrino oscillations suggests that neutrinos have finite masses. To explain the very small size of these masses, relative to those of the other standard model fermions, it is often assumed that right-handed neutrinos exist and have a very large Majorana mass term, as well as a Yukawa coupling to the lepton doublet and up-type Higgs doublet [73]. Such supersymmetric masses are theoretically equivalent to the $\mu$-term of the Higgs sector, only the challenge is greater. Now we must not only understand why these masses are not at the Planck scale, but also why they are not electroweak scale in size. Again, string theory provides a partial answer to the first question. But as in the case of the Higgs sector $\mu$ parameter these Majorana masses are presumably generated by some dynamical mechanism.

\footnotetext{
${ }^{10}$ The same is not strictly true of the first $\mu$ term mechanism: we might also wish there to be a mass term in the superpotential for right-handed neutrinos, and perhaps other states, in the low-energy theory.
} 
Proper phenomenology requires $M_{v} \sim 10^{12}-10^{16} \mathrm{GeV}$ so the Kähler potential mechanism is unlikely to prove useful. This leaves only the dynamical mechanism of generating Majorana mass terms through the trilinear coupling of right-handed neutrino bilinears to a standard model singlet which acquires a vacuum value. Unlike the Higgs mass term of the previous question, the standard see-saw mechanism demands that right-handed neutrinos have a bilinear coupling in which each species of chiral-superfield couples to itself-a type of coupling that may be forbidden by string-selection rules [74]. Furthermore, the fields which we come to identify as right-handed neutrinos may be linear combinations of fields which are standard model singlets present at string energies, making the effective Majorana mass matrix field dependent and involved. In principle such mixing between low-energy MSSM fields and string-energy exotics could generate effective Majorana mass terms for standard model non-singlets as well, which would generically be a phenomenological disaster. Thus the string theory must select the eventual right-handed neutrino superfield for special treatment:

Question 24: If right-handed neutrinos are not true singlets what are the string-theory properties of these fields that make them the only SM fields with a large supersymmetric mass?

\section{Conclusion}

The questions presented here are meant as a starting point for further inquiry. It would be unfair to say that no answers yet exist for some of these questions, but what we seek is deeper than an 'existence proof' that an answer is possible (though such a first step is lacking in some of the questions we pose). Rather, we are interested in broad statements that can be used to distinguish string theories by their phenomenological properties and which may lead to deeper understanding of how the formal properties of string constructions manifest themselves in the low-energy world of observations.

We are encouraged by the fact that so many of these questions lend themselves to a geometrical interpretation at some stage in the string construction. This suggests that a meaningful string phenomenology can be built around the classification of string theories by the properties of their moduli. To appreciate the enormous potential for progress in this area, consider the following. Moduli stabilization and spontaneous SUSY breaking and transmission tend to come together in effective field theories based on string models. This leads to a determination of the soft supersymmetry breaking Lagrangian for the observable sectorand ultimately to an understanding of patterns of masses of superpartners. But the moduli also appear in the Yukawa couplings of these theories. Their symmetries are thus potentially flavour symmetries and their vevs determine the magnitudes of these couplings (though this is somewhat more complicated if some 'matter' fields end up with vevs in the effective trilinear couplings). This leads to a pattern of flavour textures and eventually fermion masses. CP violation might enter the 4D world via both SUSY breaking operators and Yukawa couplings which are functions of these moduli, so it too emerges from this same physics. Finally, one of the string moduli is an ideal candidate for an inflation and its (above-mentioned) couplings to SM fields allows for reheating and possible baryogenesis (say through the Affleck-Dine mechanism).

Question 25: Is it a reasonable goal to imagine a theory that explains the interlocking relationships between fermion masses, the nature of dark matter, collider experiments and current cosmological observations? 
Only string theory can ever hope to provide such an all-encompassing theoretical framework. More progress will occur in this exciting area if more people work in the directions we suggest above. Our goal has been to focus increased attention on some issues where better understanding will lead to progress, and where the understanding of the theory may have reached a level allowing answers to some questions of phenomenological value.

\section{Acknowledgments}

The authors would like to thank the Aspen Center for Physics for their hospitality during the final stages of this work, as well as Vijay Balasubramanian, Joel Giedt, Burt Ovrut and many others for their input and suggestions. BN was supported by the US Department of Energy under grant No. DOE-EY-76-02-3071.

\section{References}

[1] Dienes K R and Kolda C F 1997 Twenty open questions in supersymmetric particle physics Preprint hep-ph/9712322

[2] Ellis J R 1998 Beyond the standard model for hillwalkers Preprint hep-ph/9812235

[3] Dudas E 2000 Class. Quantum Grav. 17 R41

[4] Munoz C 2003 Desperately seeking the standard model Preprint hep-ph/0312091

[5] Chung D J H, Everett L L, Kane G L, King S F, Lykken J and Wang L T 2005 Phys. Rep. 4071

[6] Abel S and Santiago J 2004 J. Phys. G: Nucl. Part. Phys. 30 R83

[7] Blumenhagen R, Cvetic M, Langacker P and Shiu G 2005 Toward realistic intersecting D-brane models Preprint hep-th/0502005

[8] Dine M 1993 Some questions of flavor in supersymmetry Preprint hep-ph/9306328

[9] Nir Y and Rattazzi R 1996 Phys. Lett. B 382363

[10] Babu K S and Mohapatra R N 1999 Phys. Rev. Lett. 832522

[11] Ross G G and Velasco-Sevilla L 2003 Nucl. Phys. B 6533

[12] Gaillard M K and Nelson B D 2000 Nucl. Phys. B 5713

[13] Carlos B de, Casas J A and Munoz C 1993 Nucl. Phys. B 399623

[14] Blanco-Pillado J J et al 2004 J. High Energy Phys. JHEP11(2004)063

[15] Marchesano F and Shiu G 2005 Phys. Rev. D 71011701

[16] Kumar J and Wells J D 2005 Phys. Rev. D 71026009

[17] Conlon J P and Quevedo F 2004 J. High Energy Phys. JHEP10(2004)039

[18] Cascales J F G and Uranga A M 2003 J. High Energy Phys. JHEP05(2003)011

[19] Marchesano F and Shiu G 2004 J. High Energy Phys. JHEP11(2004)041

[20] Dimopoulos S, Raby S and Wilczek F 1981 Phys. Rev. D 241681

[21] Dimopoulos S and Georgi H 1981 Nucl. Phys. B 193150

[22] Ibanez L E and Ross G G 1981 Phys. Lett. B 105439

[23] Boer W de and Sander C 2004 Phys. Lett. B 585276

[24] Ghilencea D M and Ross G G 2001 Nucl. Phys. B 606101

[25] Dienes K R 1997 Phys. Rep. 287447

[26] Nilles H P and Stieberger S 1997 Nucl. Phys. B 4993

[27] Blumenhagen R, Lüst D and Stieberger S 2003 J. High Energy Phys. JHEP07(2003)036

[28] Ibanez L E, Mas J, Nilles H P and Quevedo F 1988 Nucl. Phys. B 301157

[29] Mohaupt T 1994 Int. J. Mod. Phys. A 94637

[30] Cvetic M and Langacker P 2000 Nucl. Phys. B 586287

[31] Font A, Ibanez L E, Nilles H P and Quevedo F 1988 Nucl. Phys. B 307109 Font A, Ibanez L E, Nilles H P and Quevedo F 1988 Nucl. Phys. B 310764 (erratum)

[32] Cvetic M 1987 Phys. Rev. Lett. 592829

[33] Cvetic M, Everett L L, Langacker P and Wang J 1999 J. High Energy Phys. JHEP04(1999)020

[34] Witten E 1986 Nucl. Phys. B 26879

[35] Braun V, He Y H, Ovrut B A and Pantev T 2005 J. High Energy Phys. JHEP06(2005)039

[36] Braun V, He Y H, Ovrut B A and Pantev T 2005 Heterotic standard model moduli Preprint hep-th/0509051

[37] Bagger J and Poppitz E 1993 Phys. Rev. Lett. 712380 
[38] Benakli K and Smirnov A Y 1997 Phys. Rev. Lett. 794314

[39] Banks T, Grossman Y, Nardi E and Nir Y 1995 Phys. Rev. D 525319

[40] Faraggi A E 1997 Phys. Lett. B 39888

[41] Ellis J R, Faraggi A E and Nanopoulos D V 1998 Phys. Lett. B 419123

[42] Klebanov I R and Witten E 2003 Nucl. Phys. B 6643

[43] Gaillard M K 2004 R-parity from the heterotic string Preprint hep-th/0412079

[44] Carlos B de, Casas J A, Quevedo F and Roulet E 1993 Phys. Lett. B 318447

[45] Chang S, Coriano C and Faraggi A E 1996 Nucl. Phys. B 47765

[46] Benakli K, Ellis J R and Nanopoulos D V 1999 Phys. Rev. D 59047301

[47] Faraggi A E and Pospelov M 2002 Astropart. Phys. 16451

[48] Shiu G and Wang L T 2004 Phys. Rev. D 69126007

[49] Kane G L, King S F, Peddie I N R and Velasco-Sevilla L 2005 J. High Energy Phys. JHEP08(2005)083

[50] Chaudhuri S, Hockney G and Lykken J 1996 Nucl. Phys. B 469357

[51] Antoniadis I, Ellis J R, Hagelin J S and Nanopoulos D V 1989 Phys. Lett. B 23165

[52] Faraggi A E 1992 Nucl. Phys. B 387239

[53] Faraggi A E 1994 Phys. Lett. B 32662

[54] Cvetic M, Langacker P and Shiu G 2002 Phys. Rev. D 66066004

[55] Kobayashi T, Raby S and Zhang R J 2005 Nucl. Phys. B 7043

[56] Dine M, Leigh R G and MacIntire D A 1992 Phys. Rev. Lett. 692030

[57] Bailin D, Kraniotis G V and Love A 1998 Phys. Lett. B 432343

[58] Strominger A and Witten E 1985 Commun. Math. Phys. 101341

[59] Csaki C 1996 Mod. Phys. Lett. A 11599

[60] Kumar P and Lykken J D 2004 J. High Energy Phys. JHEP07(2004)001

[61] Anisimov A, Dine M, Graesser M and Thomas S 2002 Phys. Rev. D 65105011

[62] Anisimov A, Dine M, Graesser M and Thomas S 2002 J. High Energy Phys. JHEP03(2002)036

[63] Dvali G R and Pomarol A 1996 Phys. Rev. Lett. 773728

[64] Mohapatra R N and Riotto A 1997 Phys. Rev. D 554262

[65] Font A, Ibanez L E, Quevedo F and Sierra A 1990 Nucl. Phys. B 331421

[66] Giedt J 2002 Ann. Phys. 29767

[67] Cleaver G, Cvetic M, Espinosa J R, Everett L L, Langacker P and Wang J 1999 Phys. Rev. D 59055005

[68] Binetruy P, Gaillard M K and Nelson B D 2001 Nucl. Phys. B 60432

[69] Ellis J R, Enqvist K, Nanopoulos D V and Zwirner F 1986 Nucl. Phys. B 27614

[70] Cvetic M, Demir D A, Espinosa J R, Everett L L and Langacker P 1997 Phys. Rev. D 562861

Cvetic M, Demir D A, Espinosa J R, Everett L L and Langacker P 1998 Phys. Rev. D 58119905 (erratum)

[71] Giudice G F and Masiero A 1988 Phys. Lett. B 206480

[72] Antoniadis I, Gava E, Narain K S and Taylor T R 1994 Nucl. Phys. B 432187

[73] Gonzalez-Garcia M C and Nir Y 2003 Rev. Mod. Phys. 75345

[74] Giedt J, Kane G L, Langacker P and Nelson B D 2005 Phys. Rev. D 71115013 